\title{
Influence of noise shape on excitation kinetics of impurity doped quantum dots
}

\author{
Suvajit Pal $^{1}$, Sudarson Sekhar Sinha ${ }^{2}$, Jayanta Ganguly ${ }^{3}$, and Manas Ghosh ${ }^{4}$,* \\ 1 Department of Chemistry, Hetampur Raj High School, Hetampur, Birbhum 731124, West Bengal, India \\ 2 Department of Chemistry and Biochemistry, Jackson State University, Mississippi, MS 39217-0510, USA \\ 3 Department of Chemistry, Brahmankhanda Basapara High School, Basapara, Birbhum 731215, West Bengal, India \\ 4 Department of Chemistry, Physical Chemistry Section, Visva Bharati University, Santiniketan, Birbhum 731235, West Bengal, India
}

Received 2 December 2013 / Accepted 22 March 2014 / Published online 5 May 2014

\begin{abstract}
Noise influences the functioning of nanomaterials and optoelectronic materials to a significant extent. We investigate the excitation kinetics of a repulsive impurity doped quantum dot initiated by the application of external white noise with special reference to noise shape. We have exploited white noise of Gaussian and Lorentzian shapes. The said shapes in effect alter the range of spatial correlation of the white noise. We have considered both additive and multiplicative noise (in Stratonovich sense). In conjunction with the shape, the noise strength and the dopant location also fabricate the kinetics in a delicate way. Moreover, the influences of additive and multiplicative nature of the noise on the excitation kinetics have been observed to be prominently different. Interestingly as well as surprisingly enough, in case of additive noise, the noise strength does not affect the kinetics. The investigation reveals that the Lorentzian shape invites more diversities in the excitation kinetics when noise strength and dopant location are varied over a range than the Gaussian one. The Lorentzian shape causes a surge in the excitation rate over the other shape. The present investigation provides some useful perceptions of the functioning of mesoscopic devices where noise plays some profound role. Implications and influences: The present work assumes to have significance in engineering and technological applications of quantum dot nanodevices. Since in most of the devices noise plays some important role, the present study could also sincerely motivate further research on optical properties of those devices in presence of noise.
\end{abstract}

Key words: Quantum dot, Impurity, Dopant location, White noise, Noise shape, Excitation rate

$\begin{array}{ll}\begin{array}{l}\text { List of nomenclature defining the mathematical } \\ \text { symbols/variables }\end{array} \\ \text { Symbols } & \text { Nomenclature } \\ V(x, y) & \text { Harmonic confinement potential } \\ B & \text { Magnetic field } \\ \omega_{0} & \text { Harmonic confinement frequency } \\ \omega_{\mathrm{c}} & \text { Cyclotron frequency (a measure of magnetic } \\ & \text { confinement offered by } B) \\ m^{*} & \text { Effective electronic mass within the lattice of the } \\ & \text { material } \\ A & \text { Vector potential } \\ V_{0} & \text { Measure of the strength of impurity potential } \\ \gamma_{0}{ }^{-1} & \text { Spatial stretch of the impurity potential } \\ r_{0} & \text { Dopant location } \\ \zeta & \text { Noise strength } \\ \left(x_{0}, y_{0}\right) & \text { Dopant coordinate } \\ \psi(x, y) & \text { Trial wave function } \\ \phi_{n}(\alpha x), \phi_{m}(\beta y) & \text { Harmonic oscillator eigenfunctions }\end{array}$

*e-mail: pcmg77@rediffmail.com

$\begin{array}{ll}\xi(t) & \text { The noise } \\ P_{0}(t) & \text { Ground state population } \\ P_{k}(t) & \text { The population of } k \text { th state of } H_{0} \text { at time } t \\ a_{k}(t) & \text { Time-dependent superposition coefficient for the } \\ & k \text { th eigenstate }\end{array}$

\section{Introduction}

Over the last couple of decades we envisage a notable surge in theoretical and experimental researches on impurity states of low-dimensional heterostructures. Quantum dots (QD), beyond any doubt, emerge as one of the most promising mesoscopic systems out of those heterostructures. The process of miniaturization of semiconductor devices ends with QD thereby uplifting its importance. Furthermore, the typical properties of doped QDs have initiated extensive scientific study and technological applications. In doped QDs, the confinement sources undergo sensitive interplay with impurity potentials making it a prolific field of research [1]. Under the confinement, the dopant location can tailor the electronic and optical properties of the system. This resulted to a wealth of important investigations on 
impurity states [2-11, 13-15] in general, and also on their optoelectronic properties, in particular, for a wide variety of semiconductor devices [16-31]. The research trend sheds light on new device physics mingled with profound technological impact. The research trend also extends to exploring the dynamical aspects of doped QD [32, 33] giving ample importance to internal transitions between impurity induced states [34, 35]. As a result, one of the promising dynamical aspects comes out to be excitation of strongly confined electron because of its importance in opto-electronic devices and as lasers. Looking at the potential technological applications such excitation further involves optical encoding, multiplexing, photovoltaic and light emitting devices. The phenomenon also marks its signature on the population transfer among the exciton states in QD $[33,36]$.

It is quite well-known that noise in mesoscopic systems influences the functioning of the device to a great extent. As the size of the electronic system reaches the nanometer scale the role of noise becomes more and more conspicuous [37]. In practical situations, noise emerges as one of the principal deterrent in the manufacture of semiconductor heterostructure devices for diverse applications and more often than not it also restricts the level of device performance [38]. Pioro-Ladriêre et al. have also illustrated the importance of noise in self-assembled InAs QDs embedded in GaAs [39]. The noise can result externally, or it may be intrinsic. Intrinsic noises are generally the outcome of changes in impurity configurations [40]. Interestingly, low frequency noise appears as one of the prime requirements in QD heterostructures because of its potential use in a wide range of optoelectronic devices. The notable applications include industrial manufacturing, medicine, remote sensing, space communications, and military uses [41], to mention a few. Such noise measurements are also becoming useful as a pretty innocuous method for the determination of structural disorders entered during production or operation of the devices $[42,43]$. The extent of stress suffered by QD during its growth produces lattice defects which diffuse through the QD structure. The said low-frequency noise also provides a diagnostic tool for defect related properties of materials and structures [44]. Chabola and Ibrahim have extensively studied the role of lowfrequency noise in non-homogeneously doped semiconductors [42]. Of late we have made some investigations on excitation kinetics of doped QD induced by noise [45].

In the present manuscript we have emphasized on investigating the role of noise shape on excitation kinetics of doped QD. In view of this we have exploited white noises of Gaussian and Lorentzian shapes. The aforesaid shapes simply alter the range of spatial correlation of the white noise. A Gaussian noise has short-range correlation whereas the Lorentzian noise bears relatively long-range spatial correlation. The present manuscript principally focuses on how such a change in the spatial correlation of noise affects the excitation kinetics. Realizing the need of a comprehensive study in this regard we have considered both additive and multiplicative noises (in Stratonovich sense). We consider external noise which is meaningful in a variety of physical situations important for functioning of mesoscopic devices. External noise comes into play when the QD system experiences fluctuations which are not self-originating. These fluctuations can be due to a fluctuating environment or can be the result of an externally applied random force [46]. The external noise can also be regarded as an external field which drives the system. Moreover, in an external noise situation the noise parameters can be controlled externally.

In the present context the importance of dopant location in fabricating dot properties are needless to mention $[1,5,19,23$, 25]. The distance dependency of dopant illuminates promising opportunities to design QD electron dynamics in doped heterostructures. Indeed, there are some important works on off-center impurities exploiting an accurate numerical method (PMM, potential morphing method) [23, 25]. We have therefore determined the time-average excitation rate $\left(\left\langle R_{\mathrm{ex}}\right\rangle\right)$ as functions of dopant location $\left(r_{0}\right)$ and noise strength $(\zeta)$ for both the noise shapes. The investigation highlights the truly complex nature of interplay between the noise strength, dopant location, the additive and multiplicative character of the noise, and most importantly, the noise shape that sensitively modulates the excitation kinetics.

\section{2. method}

The model considers an electron subject to a harmonic confinement potential $V(x, y)$ and a perpendicular magnetic field $B$. The confinement potential assumes the form $V(x, y)=\frac{1}{2} m^{*} \omega_{0}^{2}\left(x^{2}+y^{2}\right)$, where $\omega_{0}$ is the harmonic confinement frequency. Under the effective mass approximation, the stationary states of an electron in such a system is given by the eigenstates of the Hamiltonian:

$$
H_{0}^{\prime}=\frac{1}{2 m^{*}}\left[-\mathrm{i} \hbar \nabla+\frac{e}{c} A\right]^{2}+\frac{1}{2} m^{*} \omega_{0}^{2}\left(x^{2}+y^{2}\right)
$$

$m^{*}$ is the effective electronic mass within the lattice of the material to be used. We have taken $m^{*}=0.5 m_{0}$ and set $\hbar=e=m_{0}=a_{0}=1$. This value of $m^{*}$ closely resembles Ge quantum dots $\left(m^{*}=0.55\right.$ a.u.). We have used Landau gauge $[A=(B y, 0,0)]$ where $A$ stands for the vector potential. The above Hamiltonian thus turns out to be

$$
\begin{aligned}
H_{0}^{\prime}= & -\frac{\hbar^{2}}{2 m^{*}}\left(\frac{\partial^{2}}{\partial x^{2}}+\frac{\partial^{2}}{\partial y^{2}}\right)+\frac{1}{2} m^{*} \omega_{0}^{2} x^{2} \\
& +\frac{1}{2} m^{*}\left(\omega_{0}^{2}+\omega_{c}^{2}\right) y^{2}-\mathrm{i} \hbar \omega_{c} y \frac{\partial}{\partial x},
\end{aligned}
$$

$\omega_{c}=\frac{e B}{m^{*} c}$ being the cyclotron frequency (a measure of magnetic confinement offered by $B$ ). The value of magnetic field in atomic unit corresponds to a field strength of miliTesla (mT) order. Define $\Omega^{2}=\omega_{0}^{2}+\omega_{c}^{2}$ as the effective frequency in the $y$-direction. The model Hamiltonian (cf. Eq. (1)) sensibly represents a 2-d quantum dot with a single carrier electron $[47,48]$. The form of the confinement potential conforms to kind of lateral electrostatic confinement (parabolic) of the electrons in the $x-y$ plane $[2,13,20,27,49]$.

In the present problem we have considered that the QD is doped with a repulsive Gaussian impurity [50, 51]. Introducing the impurity potential to the Hamiltonian (cf. Eq. (2)) it transforms to:

$$
H_{0}\left(x, y, \omega_{c}, \omega_{0}\right)=H_{0}^{\prime}\left(x, y, \omega_{c}, \omega_{0}\right)+V_{\text {imp }}\left(x_{0}, y_{0}\right),
$$


where $\quad V_{\text {imp }}\left(x_{0}, y_{0}\right)=V_{\text {imp }}(0)=V_{0} \mathrm{e}^{-\gamma_{0}\left[\left(x-x_{0}\right)^{2}+\left(y-y_{0}\right)^{2}\right]} \quad$ with $\gamma_{0}>0$ and $V_{0}>0$ for repulsive impurity, and $\left(x_{0}, y_{0}\right)$ denotes the coordinate of the impurity center. $V_{0}$ is a measure of the strength of impurity potential whereas $\gamma_{0}^{-1}$ determines the spatial stretch of the impurity potential. The presence of repulsive scatterer simulates dopant with excess electrons. The use of such Gaussian impurity potential is quite well-known [53-55]. Sánchez et al. [56] introduced a new confinement potential for the spherical QD's called Modified Gaussian Potential (MGP) and showed that this potential can predict the spectral energy and wave functions of a spherical quantum dot.

Thus, the problem reduces to modeling the energy eigenvalues and eigenvectors of the two dimensional Hamiltonian $H_{0}$ :

$$
H_{0} \psi_{n}(x, y)=E_{n} \psi_{n}(x, y) \text {. }
$$

Equation (2) reduces to the energy eigenvalue equation of a two dimensional harmonic oscillator as $\omega_{c}$ (i.e., $\left.B\right) \rightarrow 0$ and $V_{0} \rightarrow 0$. It would be natural therefore to seek diagonalization of $H_{0}$ in the direct product basis of harmonic oscillator eigenfunctions. Thus, the time-independent Schrödinger equation has been solved using variational method expressing the trial wave function $\psi(x, y)$ as a superposition of the product of harmonic oscillator eigenfunctions $\phi_{n}(\alpha x)$ and $\phi_{m}(\beta y)$ respectively, as

$$
\psi(x, y)=\sum_{n, m} C_{n, m} \phi_{n}(\alpha x) \phi_{m}(\beta y)
$$

where $C_{n, m}$ are the variational parameters and $\alpha=\sqrt{\frac{m^{*} \omega_{0}}{\hbar}}$ and $\beta=\sqrt{\frac{m^{*} \Omega}{\hbar}}$. The general expression for the matrix elements of $H_{0}^{\prime}$ in the chosen basis are as follows:

$$
\begin{aligned}
\left(H_{0}^{\prime}\right)_{n, m ; n^{\prime}, m^{\prime}}=\hbar\left\{\left(n^{\prime}+\frac{1}{2}\right) \omega_{0}+\left(m^{\prime}+\frac{1}{2}\right) \sqrt{\omega_{0}^{2}+\omega_{c}^{2}}\right\} \delta_{n, n^{\prime}} \delta_{m, m^{\prime}} \\
-\mathrm{i} \hbar \omega_{c} \frac{\alpha}{\beta}\left[\left\{\sqrt{\frac{n^{\prime}}{2}} \delta_{n^{\prime}-1, n}-\sqrt{\frac{n^{\prime}+1}{2}} \delta_{n^{\prime}+1, n}\right\},\right. \\
\\
\left.\left\{\sqrt{\frac{m^{\prime}+1}{2}} \delta_{m^{\prime}+1, m}+\sqrt{\frac{m^{\prime}}{2}} \delta_{m^{\prime}-1, m}\right\}\right] .
\end{aligned}
$$

In the linear variational calculation, an appreciably large number of basis functions have been exploited after making the required convergence test.

With the application of external noise the time-dependent Hamiltonian reads

$$
H(t)=H_{0}+V(t)
$$

The noise consists of random term $\langle\xi(t)\rangle$ assumed to be following Gaussian and Lorentzian distributions and characterized by the equations:

$$
\langle\xi(t)\rangle=0,
$$

the zero mean value condition, and

$$
\left\langle\xi(t) \xi\left(t^{\prime}\right)\right\rangle=2 \zeta \delta\left(t-t^{\prime}\right),
$$

the two-time correlation condition with a negligible correlation time, $\zeta$ being the noise strength. The highly fluctuating term $\xi(t)$ is called white noise because of a flat spectrum in frequency space, like that of white light. The noise strength $\zeta$ becomes simply a measure of intensity of fluctuation. We have invoked Box-Muller algorithm to generate $\xi(t)$. An additive noise term is a random term that does not depend on coordinates of system so that $V(t)=\xi(t)$. Multiplicative noise depends on the instantaneous value of the variables of the system, or more specifically on the state of the system. It does not scale with system size and is not necessarily small [46]. We thus incorporate the multiplicative noise by coupling the noise term with the system coordinates making $V(t)=\xi(t)(x+y)$ in Stratonovich sense [57]. The matrix element of $H_{0}$ due to $V(t)$ looks like

$$
\begin{aligned}
V_{p, q}^{\text {noise }}(t)= & \left\langle\psi_{p}(x, y)|V(t)| \psi_{q}(x, y)\right\rangle \\
= & \sum_{n m} \sum_{n^{\prime} m^{\prime}} C_{n m, p}^{*} C_{n^{\prime} m^{\prime}, q}\left\langle\phi_{n}(\alpha x) \phi_{m}(\beta y)\right. \\
& \left.\left|V_{2}(t)\right| \phi_{n^{\prime}}(\alpha x) \phi_{m^{\prime}}(\beta y)\right\rangle \\
= & \xi(t) \sum_{n m} \sum_{n^{\prime} m^{\prime}} C_{n m, p}^{*} C_{n^{\prime} m^{\prime}, q} \delta_{n, n^{\prime}} \delta_{m, m^{\prime}},
\end{aligned}
$$

for additive noise, and

$$
\begin{aligned}
V_{p, q}^{\text {noise }}(t)= & \left\langle\psi_{p}(x, y)|V(t)| \psi_{q}(x, y)\right\rangle \\
= & \sum_{n m} \sum_{n^{\prime} m^{\prime}} C_{n m, p}^{*} C_{n^{\prime} m^{\prime}, q}\left\langle\phi_{n}(\alpha x) \phi_{m}(\beta y)\right. \\
= & \xi(t) \sum_{n m} \sum_{n^{\prime} m^{\prime}} C_{n m, p}^{*} C_{n^{\prime} m^{\prime}, q} \\
& {\left[\frac{1}{\alpha}\left\{\sqrt{\frac{n^{\prime}+1}{2}} \delta_{n^{\prime}+1, n}+\sqrt{\frac{n^{\prime}}{2}} \delta_{n^{\prime}-1, n}\right\} \delta_{m, m^{\prime}}\right.} \\
+ & \left.\frac{1}{\beta}\left\{\sqrt{\frac{m^{\prime}+1}{2}} \delta_{m^{\prime}+1, m}+\sqrt{\frac{m^{\prime}}{2}} \delta_{m^{\prime}-1, m}\right\} \delta_{n, n^{\prime}}\right],
\end{aligned}
$$

for multiplicative noise.

The time-dependent Schrödinger equation (TDSE) containing the evolving wave function has now been solved numerically by 6 th order Runge-Kutta-Fehlberg method. In the numerical solution appropriate initial conditions have been used and numerical stability has been checked. We define the quantity $P_{k}(t)=\left|a_{k}(t)\right|^{2}$ to indicate the population of $k$ th state of $H_{0}$ at time $t$ where $a_{k}(t)$ is the time-dependent superposition coefficient for the $k$ th eigenstate of the unperturbed Hamiltonian. Actually $P_{k}(t)$ is the probability of observing the electron in the $k$ th eigenstate and is therefore given by modulus square of the time-dependent superposition coefficients. We observe a continuous variation in the ground state population $\left[P_{0}(t)\right]$ during the time evolution. Naturally the quantity $Q(t)=1-P_{0}(t)$ serves as a measure of excitation. In consequence, the quantity $R_{\mathrm{ex}}(t)=\frac{\mathrm{d} Q}{\mathrm{~d} t}$ serves as the time-dependent rate of excitation from which the time-average rate of excitation $\left[\left\langle R_{\mathrm{ex}}\right\rangle=\frac{1}{T} \int_{0}^{T} R_{\mathrm{ex}}(t) \mathrm{d} t\right]$ can be calculated where $T$ is the total time of dynamic evolution [45].

\section{Results and discussion}

\subsection{Analysis of additive noise}

In order to analyze the exclusive role played by the dopant location on excitation kinetics, we have plotted $\left\langle R_{\mathrm{ex}}\right\rangle$ as a 


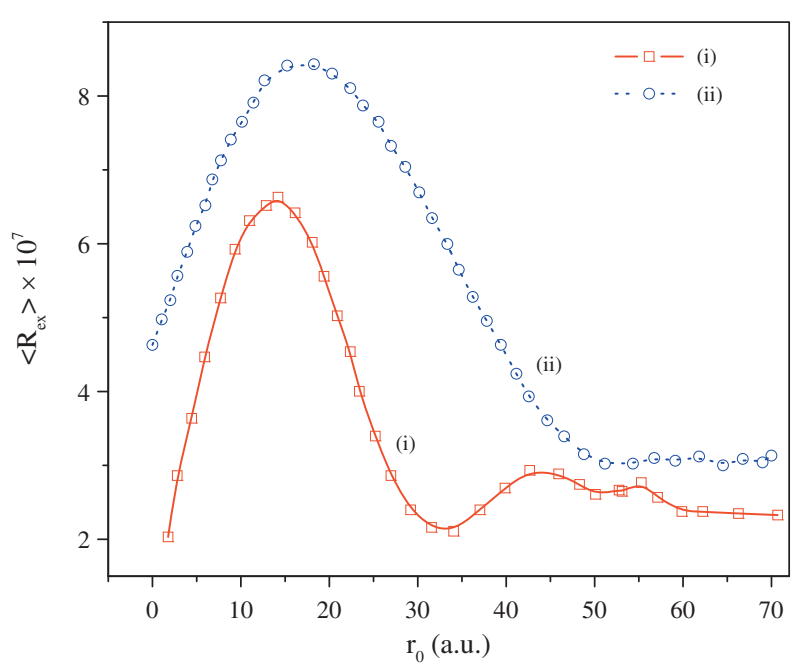

Figure 1. Plot of $\left\langle R_{\mathrm{ex}}\right\rangle$ vs. $r_{0}$ for additive white noise of fixed strength $\zeta=1.0 \times 10^{-6}$ a.u.: (i) Gaussian and (ii) Lorentzian.

function of dopant location $\left(r_{0}\right)$ for fixed value of noise strength $\left(\zeta=1.0 \times 10^{-6}\right.$ a.u.) for both Gaussian and Lorentzian white noises (Figure 1). Qualitatively, both the plots exhibit similar kinetics consisting of a maxima at some typical dopant location and saturation at far off-center positions. The maximization takes place at $r_{0} \sim 14.0$ a.u. and $r_{0} \sim 17.0$ a.u. for Gaussian and Lorentzian noises, respectively. It needs to be realized that the role of dopant coordinate on excitation rate is not at all straightforward, rather it is quite delicate. An increase in $r_{0}$ takes the dopant away from the confines of dot potential favoring excitation. However, the said shift also reduces dot-impurity repulsive interaction thereby suppressing excitation. Thus, a progressive shift of dopant to more and more off-center positions invites two opposite factors come into play that can affect excitation rate. It is the changeover of relative dominance of one of the contrasting factors over the other that causes maximization in the kinetics at some typical dopant locations. The occurrence of saturation in kinetics at far off-center locations indicates sort of compromise between the diverse factors that affect excitation. The change in noise shape from Gaussian to Lorentzian simply shifts the maxima to some more off-center location. This in turn implies that the long-range correlation associated with the Lorentzian noise promotes excitation to a maximum when the dot-impurity separation is larger than in case of Gaussian noise. From Figure 1 it also comes out that the Lorentzian noise causes an overall increase in the excitation rate over that of Gaussian counterpart throughout the entire range of dopant incorporation. The observations suggest that Lorentzian noise causes greater perturbation to the system over the other one. It remains interesting to enquire whether the same conclusion can be drawn in case of multiplicative noise.

Surprisingly enough, in the present case we do not envisage any influence of noise strength $(\zeta)$ on the excitation kinetics. The reluctance of noise strength to influence the excitation is interesting and presumably originates from its very "additive" nature. It seems that, since the additive noise remains uncoupled to the system coordinates and simply adds on to the Hamiltonian, it perturbs all the eigenstates to equal extents. Thus, the

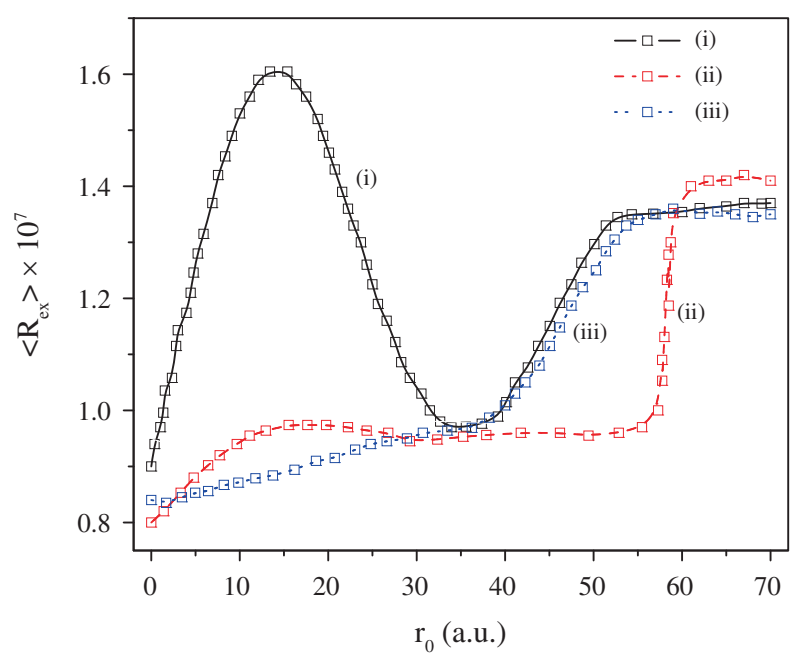

Figure 2a. Plot of $\left\langle R_{\mathrm{ex}}\right\rangle$ vs. $r_{0}$ for Gaussian multiplicative white

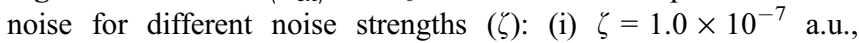
(ii) $\zeta=1.0 \times 10^{-5}$ a.u., and (iii) $\zeta=1.0 \times 10^{-4}$ a.u.

relative separations between all the eigenstates remain unaltered making the excitation kinetics reticent to the noise strength. This observation further necessitates investigation of the role of multiplicative noise in the present context.

\subsection{Analysis of multiplicative noise}

Insofar as excitation is concerned, coupling the noise term to the system coordinate is expected to bring about more subtle interplay between the dot confinement and the noise strength. Already, in the context of Figure 1, we have discussed how different factors inherently affect the excitation kinetics in contrasting ways as the dopant is shifted to more and more offcenter locations. However, in addition to the dopant location, now we have to take into account the contribution from $\zeta$ also. It is expected that by virtue of its intrinsic characteristic the noise is likely to promote excitation. In what follows, we discuss the modulation of excitation kinetics by multiplicative Gaussian and Lorentzian noise with special reference to dopant location and noise strength.

\subsubsection{Role of dopant location $\left(r_{0}\right)$}

\section{(I) Gaussian noise}

Figure 2a delineates $\left\langle R_{\mathrm{ex}}\right\rangle$ as a function of dopant location for different values of $\zeta$. From Figure $2 \mathrm{a}$ we notice that at low noise strength $\left(\zeta=1.0 \times 10^{-7}\right.$ a.u.), the excitation rate passes through a maxima at $r_{0} \sim 14.0$ a.u. (nearly at the same dopant location where we have observed maximization in case of additive noise, Figure 1). However, no such maximization is observed using multiplicative noise of medium $(\zeta=1.0 \times$ $10^{-5}$ a.u. $)$ and high $\left(\zeta=1.0 \times 10^{-4}\right.$ a.u. $)$ strength. It is also evident from the figure that for all noise strengths the excitation rate attains steady value at far off-center dopant locations. A multiplicative noise of extremely low strength perturbs the system feebly and hardly leaves its signature on excitation kinetics. Thus, the excitation kinetics effectively depends on the dopant location and exhibits similar pattern as that of additive noise. 
As the noise strength assumes some intermediate value $\left(\zeta=1.0 \times 10^{-5}\right.$ a.u.), the rate profile after some small initial rise exhibits a steady behavior within the range 15.7 a.u. $\leq r_{0} \leq 55.5$ a.u.. Beyond $r_{0} \geq 55.5$ a.u. the excitation rate jumps abruptly to a high value and permanently settles to steady value beyond $r_{0} \geq 63.1$ a.u. Such rate profile reveals the nuances of combined influence of several factors that affect excitation. The initial small rise in $\left\langle R_{\text {ex }}\right\rangle$ at near off-center locations $\left(r_{0} \leq 15.7\right.$ a.u.) indicates mild win of noise and dot-impurity repulsive terms over that of strong confinement potential. After that there is a sustained balance between the diverse factors that affect excitation over a quite extended range of dopant location (15.7 a.u. $\leq r_{0} \leq 55.5$ a.u.). At $r_{0}=55.5$ a.u., the confinement becomes quite weak and factors that promote excitation (noise + repulsive interaction) suddenly become prominent leading to an abrupt rise in excitation rate. Beyond $r_{0} \geq 63.1$ a.u., because of large dot-impurity separation, their mutual repulsive interaction gets considerably diminished and we land up at a permanent balanced condition of those factors revealed through a saturation in excitation kinetics. With a high value of noise strength $\left(\zeta=1.0 \times 10^{-4}\right.$ a.u. $)$ the rate profile manifests kind of resemblance with that of medium noise strength counterpart. However, the variation in the rate with dopant location appears much less sharp. The initial rise of $\left\langle R_{\mathrm{ex}}\right\rangle$ occurs over an extended region $(0.0$ a.u. $\leq$ $r_{0} \leq 35.0$ a.u.) in an indolent manner, the former extended zone of constant excitation rate becomes nearly absent and the rate begins to rise as $r_{0} \geq 35.0$ a.u. From the figure it is also quite discernible that the rise in excitation rate within 35.0 a.u. $\leq r_{0} \leq 56.6$ a.u. becomes much less abrupt in comparison with the medium noise strength counterpart. The regime of persistent saturation in excitation kinetics begins from $r_{0}=56.6$ a.u. Thus, an increase in noise strength from medium to high value does not much alter the intrinsic nature of interplay between itself, the dot confinement potential and the dotdopant repulsive interaction (the last two factors undergo change with a variation in dopant location) toward fabricating the excitation kinetics. The said increase seems to disseminate the complex interplay over a spatially more spread out domain making the overall variation of the excitation rate rather sluggish with change in dopant coordinate.

\section{(II) Lorentzian noise}

A change in noise shape has been found to bring about new characteristics in the excitation kinetics. Figure $2 b$ delineates $\left\langle R_{\mathrm{ex}}\right\rangle$ as a function of dopant location for different values of $\zeta$. The figure shows that at low noise strength $\left(\zeta=1.0 \times 10^{-7}\right.$ a.u. $)\left\langle R_{\text {ex }}\right\rangle$ passes through a minima at $r_{0} \sim 19.0$ a.u. A distinct maxima in $\left\langle R_{\mathrm{ex}}\right\rangle$, however, has been observed with moderate noise strength $\left(\zeta=5.0 \times 10^{-7}\right.$ a.u. $)$. A large value of noise strength $\left(\zeta=2.25 \times 10^{-6}\right.$ a.u. $)$ causes successive maximization and minimization in excitation kinetics at $r_{0} \sim 27.0$ a.u. and $r_{0} \sim 42.0$ a.u., respectively. The kinetics exhibits saturation for far off-center dopants with all values of noise strength reflecting kind of negotiation between diverse factors that influence it. In the weak noise limit, the noise plays the second fiddle and the kinetics is mainly guided by relative strengths of dot confinement and dot-impurity repulsive interaction. In this limit the initial drop in $\left\langle R_{\mathrm{ex}}\right\rangle$ to a minimum with

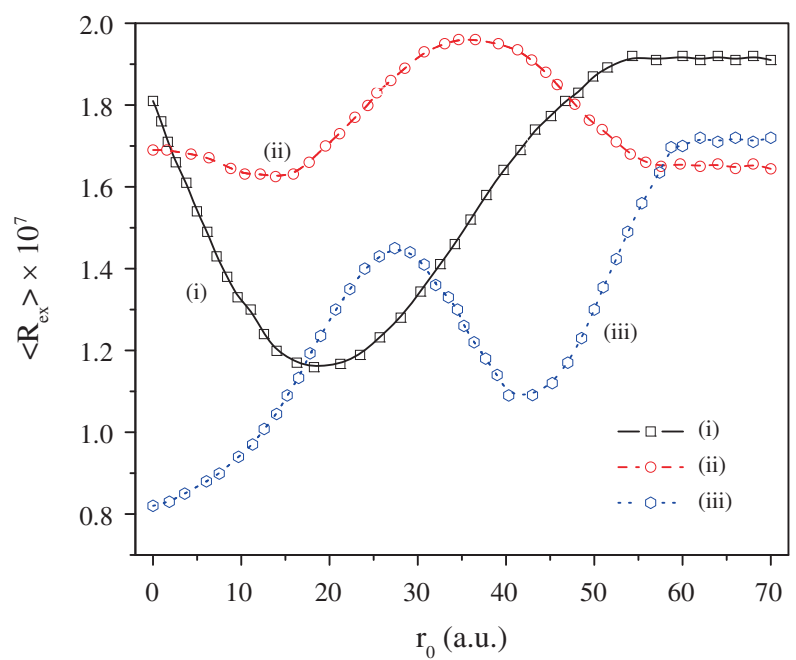

Figure 2b. Plot of $\left\langle R_{\mathrm{ex}}\right\rangle$ vs. $r_{0}$ for Lorentzian multiplicative white

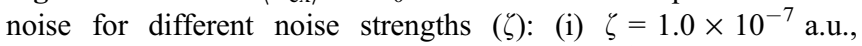
(ii) $\zeta=5.0 \times 10^{-7}$ a.u., and (iii) $\zeta=2.25 \times 10^{-6}$ a.u.

increase in $r_{0}$ could be attributed to decreasing dot-impurity overlap. As soon as $r_{0} \geq 19.0$ a.u. (the place of minimum excitation rate), the dot-impurity separation makes the dot confinement substantially quenched. Taking advantage of it, the combined effect of dot-impurity repulsive interaction and noise promotes excitation until there is saturation. A noise of moderate strength changes the scenario completely displaying maximization in kinetics. In this case, in alliance with noise, the dot-impurity repulsive interaction outshine the dot confinement resulting in said maximization. A large value of noise strength seems to cause changeover in the relative strengths of the factors that promote and impede excitation in succession. This is revealed through emergence of consecutive maximization and minimization in the excitation rate. We thus notice that a change in the shape of noise from Gaussian to Lorentzian makes the excitation rate more delicately dependent on dopant location. Whereas in case of Gaussian noise the $r_{0}$-dependence of kinetics remains more or less similar at moderate and high noise strengths, with Lorentzian noise the said dependence exhibits noticeable diversities as noise strength is increased from a rather low to a high value.

\subsubsection{Role of noise strength $(\zeta)$}

\section{(I) Gaussian noise}

Figure 2c demonstrates the variation of $\left\langle R_{\mathrm{ex}}\right\rangle$ with $\zeta$ at oncenter $\left(r_{0}=0.0\right.$ a.u. $)$ and two off-center $\left(r_{0}=28.28\right.$ a.u. and 70.71 a.u.) dopant locations. Such a demonstration focuses mainly on the role played by the noise strength in shaping the excitation kinetics. Figure $2 \mathrm{c}$ evinces that, more or less at all dopant locations, $\left\langle R_{\mathrm{ex}}\right\rangle$ passes through a maximum for some typical values of noise strength depending upon the dopant location. Moreover, the kinetics terminates to some saturation at high values of noise strength. The appearance of maxima can as usually be conceived on the basis of changeover of relative dominance of parameters having contrasting influences on excitation kinetics. The changeover is actually associated with the variation of noise strength. It is also interesting to note from 


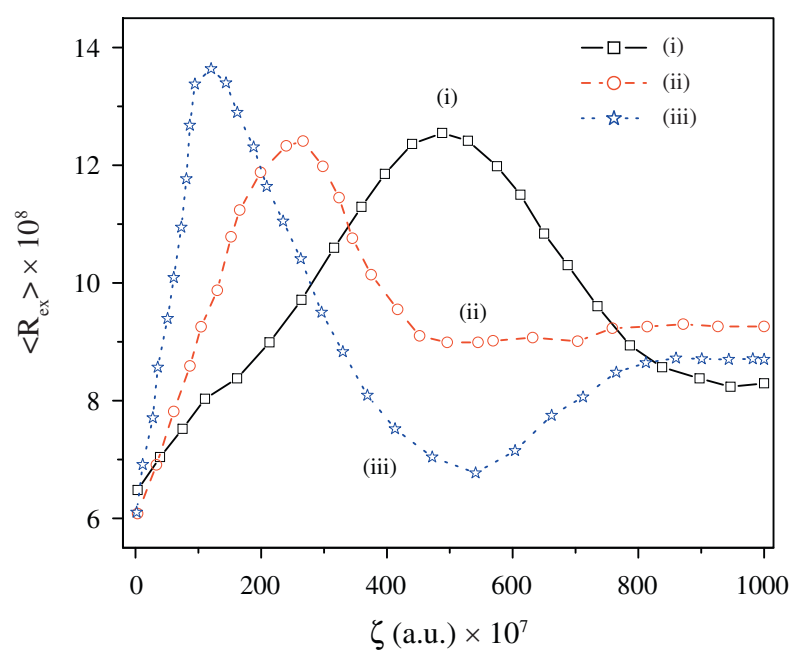

Figure 2c. Plot of $\left\langle R_{\mathrm{ex}}\right\rangle$ vs. $\zeta$ for Gaussian multiplicative white noise for different dopant locations $\left(r_{0}\right)$ : (i) $r_{0}=0.0$ a.u., (ii) $r_{0}=28.28$ a.u., and (iii) $r_{0}=70.71$ a.u.

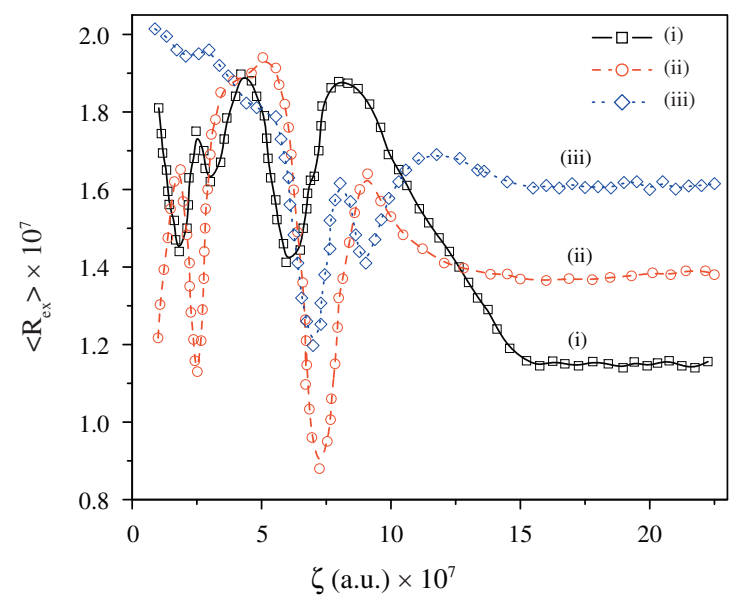

Figure 2d. Plot of $\left\langle R_{\mathrm{ex}}\right\rangle$ vs. $\zeta$ for Lorentzian multiplicative white noise for different dopant locations $\left(r_{0}\right)$ : (i) $r_{0}=0.0$ a.u., (ii) $r_{0}=28.28$ a.u., and (iii) $r_{0}=70.71$ a.u.

the figure that as the dopant is shifted from on to more and more off-center locations the emergence of maxima occurs at lower $\zeta$ values viz. at $\zeta=4.87 \times 10^{-5}$ a.u. for $r_{0}=0.0$ a.u., $\zeta=2.65 \times 10^{-5}$ a.u. for $r_{0}=28.28$ a.u., and $\zeta=1.17$ $\times 10^{-5}$ a.u. for $r_{0}=70.70$ a.u. It seems that, since such a shift of the dopant takes it away from dot confinement center, the noise becomes progressively more prompt in bringing about the changeover of parameters that affect excitation kinetics. The emergence of maxima thus occurs at more and more low values of noise strength.

\section{(II) Lorentzian noise}

Finally we discuss the role of noise strength on excitation kinetics as the noise assumes a Lorentzian shape. Figure $2 \mathrm{~d}$ delineates the variation of $\left\langle R_{\mathrm{ex}}\right\rangle$ with $\zeta$ at on-center $\left(r_{0}=0.0\right.$ a.u. $)$ and two off-center $\left(r_{0}=28.28\right.$ a.u. and 70.71 a.u.) dopant locations. The change in noise shape manifestly affects the rate profile. Here also, as in case of Gaussian noise, the profile of excitation kinetics appears quite similar at all dopant locations. However, the very pattern of rate profile is drastically affected. Unlike previous case, here we do not envisage any well-defined maximization in the kinetics at some typical noise strengths. Now, within low and moderate $\zeta$ regimes $\left(\zeta \leq 1.2 \times 10^{-6}\right.$ a.u.), the kinetics appears highly irregular comprising of lots of ups and downs indicating excitation and deexcitation in tandem. Thus, the change in noise shape from Gaussian to Lorentzian makes the kinetics haphazard when the noise strength is not very large. This simply means that, from a more fundamental perspective, the said change in shape in effect increases the frequency of changeover of the relative dominance of several factors that control the kinetics in disparate ways. It is the high $\zeta$ domain where we again observe saturation in the kinetics as in the previous case. The change in shape also marks its signature on the magnitudes of excitation rates. In fact, $\left\langle R_{\mathrm{ex}}\right\rangle$ increases by an order of magnitude for Lorentzian noise (Figure 2d) over that of Gaussian one (Figure 2c).

From an experimental perspective there exist a variety of physical situations in which external noise can be realized and assumes interest. In these situations the system is endowed with fluctuations which are not self-originating. These fluctuations can be due to a fluctuating environment or can be the outcome of an externally applied random force. Thus, the external noise can be viewed as an external field which drives the system [46]. Specifically, it may be an external light with fluctuating amplitude giving rise to noise with special characteristics of Gaussian noise. In practice, external noise can be generated by using a function generator (Hewlett-Packard 33120A) and its essential characteristics, Gaussian distribution and zero mean can be accomplished [56]. The external noise could be introduced multiplicatively using a circuit that drives the nonlinear element by using the voltage from an external source [57].

\section{Conclusions}

The excitation kinetics of impurity doped quantum dots insisted by Gaussian white noise reveals interesting dependence on noise shape. Actually, the change in shape causes a change in the extent of spatial correlation of noise which affects the kinetics. In addition to this, the interplay between the noise strength and the dopant location delicately modulates the kinetics. In case of additive noise the Lorentzian shape causes an overall hike in the kinetics over that of Gaussian shape. Moreover, the Lorentzian noise delays the onset of maximization of excitation rate by relegating it to more off-center location. However, in case of additive noise we have found the noise strength remaining indifferent toward the excitation rate. The interplay between dopant location and noise strength becomes more subtle in case of multiplicative noise. The subtlety becomes even more pronounced by applying Lorentzian noise instead of a Gaussian one. This is revealed through a more diverse dependence of kinetics on dopant location in case of Lorentzian noise than in the other. The effect of noise shape is further illustrated on examining the kinetics as a function of noise strength. Now the Lorentzian noise induces irregularities in kinetics, specially 
in low and medium noise strength domain, and also enhances the magnitude of average excitation rate over its Gaussian counterpart. On all occasions the appearance of maxima/minima in excitation kinetics indicates preponderance of the parameters that favor or inhibit excitation over the rival ones, and the saturation can be attributed to kind of compromise between them. We didn't rely upon the intuitive idea that application of noise will disrupt any profile of kinetics beyond recognition. In consequence, what we have found is that the results of investigation are interesting and expected to convey important insights in the researches on quantum dot nanomaterials and optoelectronic materials.

The present investigation seems to unfold promising opportunities for future development and research. It is possible to collect crucial information regarding charge fluctuations in the mesoscopic system through the intrusion of time-dependent noise. It will also be meaningful to study the correlations in the transport processes through nanostructures by exploiting noise.

Acknowledgements. The authors S. P., J. G. and M. G. thank D. S. T-F. I. S. T (Govt. of India) and U. G. C.-S. A. P (Govt. of India) for partial financial support.

\section{References}

1. J.L. Movilla, J. Planelles, Off-centering of hydrogenic impurities in quantum dots, Phys. Rev. B 71 (2005) 075319.

2. B. Gülveren, Ü. Atav, M. Sahin, M. Tomak, A parabolic quantum dot with $N$ electrons and an impurity, Physica E 30 (2005) 143-149.

3. E. Räsänen, J. Könemann, R.J. Puska, M.J. Haug, R.M. Nieminen, Impurity effects in quantum dots: toward quantitative modeling, Phys. Rev. B 70 (2004) 115308.

4. M. Aichinger, S.A. Chin, E. Krotscheck, E. Räsänen, Effects of geometry and impurities on quantum rings in magnetic fields, Phys. Rev. B 73 (2006) 195310.

5. W. Xie, Binding energy of an off-center hydrogenic donor in a spherical Gaussian quantum dot, Physica B 403 (2008) 2828-2831.

6. F.J. Betancur, J. Sierra-Ortega, R.A. Escorcia, J.D. González, I.D. Mikhailov, Density of impurity states in doped spherical quantum dots, Physica E 23 (2004) 102-107.

7. F.J. Betancur, I.D. Mikhailov, L.E. Oliveira, Shallow donor states in GaAs - $(\mathrm{Ga}, \mathrm{Al})$ As quantum dots with different potential shapes, J. Phys. D: Appl. Phys. 31 (1998) 3391-3396.

8. U. Yesilgul, S. Sakiroğlu, E. Kasapoglu, H. Sari, I. Sökmen, Hydrogenic impurities in quantum dots under intense high frequency laser field, Physica B 406 (2011) 1441-1444.

9. H. Taş, M. Şahin, The electronic properties of core/shell/well/ shell spherical quantum dot with and without a hydrogenic impurity, J. Appl. Phys. 111 (2012) 083702.

10. S.-S. Li, J.-B. Xia, Binding energy of a hydrogenic donor impurity in a rectangular parallelepiped-shaped quantum dot: quantum confinement and Stark effects, J. Appl. Phys. 101 (2007) 093716.

11. E. Kasapoglu, H. Sari, I. Sökmen, Density of impurity states of hydrogenic impurities in an inverse parabolic quantum well under the magnetic field, Physica B 392 (2007) 213-216.
12. S. Akgŭl, M. Şahin, K. Köksal, A detailed investigation of the electronic properties of a multi-layer spherical quantum dot with a parabolic confinement, J. Lumin. 132 (2012) 1705-1713.

13. R. Khordad, Hydrogenic donor impurity in a cubic quantum dot: effect of position-dependent effective mass, Eur. Phys. J. B 85 (2012) 114.

14. M. Cristea, E.C. Niculescu, Hydrogenic impurity states in CdSe/ $\mathrm{ZnS}$ and $\mathrm{ZnS} / \mathrm{Cdse}$ core-shell nanodots with dielectric mismatch, Eur. Phys. J. B 85 (2012) 191.

15. W. Xie, Linear and nonlinear optical properties of a hydrogenic donor in spherical quantum dots, Physica B 403 (2008) 43194322.

16. W. Xie, Impurity effects on optical property of a spherical quantum dot in the presence of an electric field, Physica B 405 (2010) 3436-3440.

17. W. Xie, Nonlinear optical properties of a hydrogenic donor quantum dot, Phys. Lett. A 372 (2008) 5498-5500.

18. W. Xie, Optical properties of an off-center hydrogenic impurity in a spherical quantum dot with Gaussian potential, Superlattices Microstruct. 48 (2010) 239-247.

19. A.J. Peter, Polarizabilities of shallow donors in spherical quantum dots with parabolic confinement, Phys. Lett. A 355 (2006) 59-62.

20. K.M. Kumar, A.J. Peter, C.W. Lee, Optical properties of a hydrogenic impurity in a confined $\mathrm{Zn}_{1-\mathrm{x}} \mathrm{Cd}_{\mathrm{x}} \mathrm{Se} / \mathrm{ZnSe}$ spherical quantum dot, Superlattices Microstruct. 51 (2012) 184-193.

21. R. Khordad, Diamagnetic susceptibility of hydrogenic donor impurity in a $V$-groove $\mathrm{GaAs} / \mathrm{Ga}_{1-x} \mathrm{Al}_{x} \mathrm{As}$ quantum wire, Eur. Phys. J. B 78 (2010) 399-404.

22. S. Baskoutas, E. Paspalakis, A.F. Terzis, Electronic structure and nonlinear optical rectification in a quantum dot: effects of impurities and external electric field, J. Phys.: Cond. Matter 19 (2007) 395024.

23. I. Karabulut, S. Baskoutas, Linear and nonlinear optical absorption coefficients and refractive index changes in spherical quantum dots: Effects of impurities, electric field, size, and optical intensity, J. Appl. Phys. 103 (2008) 073512.

24. I. Karabulut, S. Baskoutas, Second and third harmonic generation susceptibilities of spherical quantum dots: effects of impurities, electric field and size, J. Comput. Theor. Nanosci. 6 (2009) 153-156.

25. B. Çakir, Y. Yakar, A. Özmen, M. Özgür Sezer, M. Şahin, Linear and nonlinear optical absorption coefficients and binding energy of a spherical quantum dot, Superlattices Microstruct. 47 (2010) 556-566.

26. Y. Yakar, B. Çakir, A. Özmen, Calculation of linear and nonlinear optical absorption coefficients of a spherical quantum dot with parabolic potential, Opt. Commun. 283 (2010) 17951800.

27. C.A. Duque, M.E. Mora-Ramos, E. Kasapoglu, F. Ungan, U. Yesilgul, S. Sakiroglu, H. Sari, I. Sökmen, Impurity-related linear and nonlinear optical response in quantum-well wires with triangular cross section, J. Lumin. 143 (2013) 304-313.

28. S. Baskoutas, E. Paspalakis, A.F. Terzis, Effects of excitons in nonlinear optical rectification in semiparabolic quantum dots, Phys. Rev. B 74 (2006) 153306.

29. C. Xia, Z. Zeng, S. Wei, Electric field effects on optical properties in zinc-blende InGaN/GaN quantum dot, J. Lumin. 131 (2011) 623-627.

30. M. Şahin, K. Köksal, The linear optical properties of a multishell spherical quantum dot of a parabolic confinement for cases 
with and without a hydrogenic impurity, Semicond. Sci. Technol. 27 (2012) 125011.

31. E. Paspalakis, C. Simserides, S. Baskoutas, A.F. Terzis, Electromagnetically induced population transfer between two quantum well subbands, Physica E 40 (2008) 1301-1304.

32. E. Paspalakis, A. Kalini, A.F. Terzis, Local field effects in excitonic population transfer in a driven quantum dot system, Phys. Rev. B 73 (2006) 073305.

33. C.A. Duque, N. Porras-Montenegro, Z. Barticevic, M. Pacheco, L.E. Oliveira, Electron-hole transitions in self-assembled InAs/ GaAs quantum dots: effects of applied magnetic fields and hydrostatic pressure, Microelectron. J. 36 (2005) 231-233.

34. C.A. Duque, N. Porras-Montenegro, Z. Barticevic, M. Pacheco, L.E. Oliveira, Effects of applied magnetic fields and hydrostatic pressure on the optical transitions in self-assembled InAs/GaAs quantum dots, J. Phys.: Cond. Matter 18 (2006) 1877-1884.

35. E. Paspalakis, A.F. Terzis, Proceedings of the 5th WSEAS International Conference on Microelectronics, Nanoelectronics, Optoelectronics, Prague, Czech Republic, March 12-14, 2006.

36. H.K. Zhao, Shot noise in the hybrid systems with a quantum dot coupled to normal and superconducting leads, Phys. Lett. A 299 (2002) 262-270.

37. N.A. Hastas, C.A. Dimitriadis, L. Dozsa, E. Gombia, S. Amighetti, P. Frigeri, Low frequency noise of GaAs Schottky diodes with embedded InAs quantum layer and self-assembled quantum dots, J. Appl. Phys. 93 (2003) 3990-3994.

38. N.A. Hastas, C.A. Dimitriadis, L. Dozsa, E. Gombia, R. Mosca, Investigation of single electron traps induced by InAs quantum dots embedded in GaAs layer using the low-frequency noise technique, J. Appl. Phys. 96 (2004) 5735-5739.

39. M. Pioro-Ladriêre, J.H. Davies, A.R. Long, A.S. Sachrajda, L. Gaudreau, P. Zawadzki, J. Lapointe, J. Gupta, Z. Wasilewski, S. Studenikin, Origin of switching noise in $\mathrm{GaAs} / \mathrm{Al}_{x} \mathrm{Ga}_{1-x} \mathrm{As}$ lateral gated devices, Phys. Rev. B 72 (2005) 115331.

40. P. Yuan, O. Baklenov, H. Nie, A.L. Holmes Jr., B.G. Streetman, J.C. Campbell, High-speed and low-noise, IEEE J. Select. Top. Quantum Electron. 6 (2000) 422-425.

41. H.V. Asriyan, F.V. Gasparyan, V.M. Aroutiounian, S.V. Melkonyan, P. Soukiassian, Low-frequency noise in nonhomogeneously doped semiconductor, Sens. Actuators A 113 (2004) 338-343.

42. Z. Chabola, A. Ibrahim, Noise and scanning by local illumination as reliability estimation for silicon solar cells, Fluc. Noise Lett. 1 (2001) L21-L26.
43. J.I. Lee, H.D. Nom, W.J. Choi, B.Y. Yu, J.D. Song, S.C. Hong, S.K. Noh, A. Chovet, Low frequency noise in GaAs structures with embedded $\operatorname{In}(\mathrm{Ga})$ As quantum dots, Curr. Appl. Phys. 6 (2006) 1024-1029.

44. S. Pal, S.S. Sinha, J. Ganguly, M. Ghosh, Excitation kinetics of impurity doped quantum dot driven by Gaussian white noise: Interplay with external field, Chem. Phys. 426 (2013) 54-58.

45. J.M. Sancho, M.S. Miguel, S.L. Katz, J.D. Gunton, Analytical and numerical studies of multiplicative noise, Phys. Rev. A 26 (1982) 1589-1609.

46. L. Jacak, P. Hawrylak, A. Wojos, Quantum Dots, SpringerVerlag, Berlin, 1998.

47. T. Chakraborty, Quantum Dots - a survey of the properties of artificial atoms, Elsevier, Amsterdam, 1999.

48. S. Baskoutas, A.F. Terzis, E. Voutsinas, Binding energy of donor states in a quantum dot with parabolic confinement, J. Comput. Theor. Nanosci. 1 (2004) 317-321.

49. V. Halonen, P. Hyvönen, P. Pietiläinen, T. Chakraborty, Effects of scattering centers on the energy spectrum of a quantum dot, Phys. Rev. B 53 (1996) 6971-6974.

50. V. Halonen, P. Pietilinen, T. Chakraborty, Optical-absorption spectra of quantum dots and rings with a repulsive scattering centre, Europhys. Lett. 33 (1996) 337-382.

51. J. Adamowski, A. Kwaśniowski, B. Szafran, LO-phononinduced screening of electron-electron interaction in $\mathrm{D}^{-}$centres and quantum dots, J. Phys: Cond. Matter 17 (2005) 4489-4500.

52. S. Bednarek, B. Szafran, K. Lis, J. Adamowski, Modeling of electronic properties of electrostatic quantum dots, Phys. Rev. B 68 (2003) 155333.

53. B. Szafran, S. Bednarek, J. Adamowski, Parity symmetry and energy spectrum of excitons in coupled self-assembled quantum dots, Phys. Rev. B 64 (2001) 125301.

54. A. Gharaati, R. Khordad, A new confinement potential in spherical quantum dots: modified Gaussian potential, Superlattices Microstruct. 48 (2010) 276-287.

55. J. García-Ojalvo, J.M. Sancho, Noise in spatially extended systems, Springer, New York, USA, 1999.

56. E. Sánchez, M.A. Matías, V. Pérez-Muñuzuri, Analysis of synchronization of chaotic systems by noise: an experimental study, Phys. Rev. E 56 (1997) 4068-4071.

57. V. Pérez-Muñuzuri, M.N. Lorenzo, Experimental improvement of chaotic synchronization due to multiplicative time-correlated Gaussian noise, Int. J. Bifurc. Chaos 09 (1999) 2321-2327.

Cite this article as: Pal S, Sinha SS, Ganguly J \& Ghosh M: Influence of noise shape on excitation kinetics of impurity doped quantum dots. Manufacturing Rev. 2014, 1, 3. 